\title{
TEXTSORTENKRITERIEN \\ FÜR WERBEMATERIALIEN TECHNISCHER UNTERNEHMEN. TEXTTYP: TECHNICAL PROSPECT
}

\author{
KATARZYNA WIELIŃSKA \\ Adam Mickiewicz University - Poznan
}

ABSTRACT. The paper refers to the basic problems of classification of technical prospects to particular text types. On the basis of three different prospects the author makes an attempt of describing some of classifications methods and factors relevant for translation orientated text analysis. Defining conditions of classification of this popular text type can prove to be helpful for its future translators.

\section{Einleitung}

In kaum einem Lebensbereich werden heutzutage so viele Übersetzungen wie in der Technik benötigt. Der Unternehmenserfolg am Markt wird durch seine Fähigkeit zu Innovationen bestimmt. Die Neuheiten müssen jedoch schnell an die potentiellen Kunden gelangen, damit man den Wettbewerbern immer einen Schritt im voraus ist. Aus diesem Grunde gehören Übersetzer und Dolmetscher von fachsprachlichen Texten in immer höherem Maße zu Mitgliedern internationaler Kongresse im wirtschaftlichen, industriellen und wissenschaftlichen Bereich. Ungeachtet der verbreiteten Meinung, dass eine technische Übersetzung von jedem gemacht werden kann, der Fachwissen besitzt und über ein Minimum an Sprachkenntnis verfügt, sind sowohl sprachliche als auch technische Kenntnisse Voraussetzung, um fachsprachliche Übersetzungen professionell zu fertigen.

\section{Die Bedeutung von Analyse der fachsprachlichen Textsorten}

Innerhalb fachsprachlicher Textsorten gibt es eine, die für Übersetzer schwieriger als ein rein informativer Fachtext sein kann. Es handelt sich um Werbetexte technischer Unternehmen. Das Ziel der vorliegenden Studie ist ein Versuch, diese Textsorte näher zu beschreiben und zu prüfen, ob in Werbetexten von verschiedenen technischen Unternehmen Ähnlichkeiten in bezug auf Textinhalt und -aufbau 
festzustellen sind. Als Beispiel wird hier ein Prospekttext herangeführt und im Hinblick auf die Faktoren analysiert, die eine Klassifizierung des Textes zu bestimmter Textsorte erlauben.

Für die erfolgreiche Übersetzung von fachsprachlichen Werbeprospekten sind außer sprachlichen und fachlichen Kenntnisse auch andere Fähigkeiten Voraussetzung, wie z.B. den textimmanenten Appell richtig zu entschlüsseln und in der Zielsprache äquivalent wiederzugeben. Die linguistische Analyse von Beispielen dieser Textsorte und die Untersuchung der interlingualen Übersetzungsäquivalenz kann künftigen Übersetzern wichtige Hilfe leisten. Die genaue Beschreibung der Textgattung, zu der ein Textexemplar gehört, wird oft als Hauptfaktor genannt, der die verwendeten Übersetzungsprinzipien und -Methoden bestimmt.

Das Interesse für Texte als Gegenstand fachsprachlich orientierter Untersuchungen war Folge der pragmatischen Wende gegen Mitte der 70er Jahre; damals ist es auch zu einer Gegenstandserweiterung in der Fachsprachenforschung gekommen. Heutzutage wird hauptsächlich die Funktion von Texten untersucht, die Kommunikation im fachlichen Bereich gewährleisten. Vor der Wende erkannten Sprachwissenschaftler und Fachleute im Wortschatz die Komponente, die die Besonderheit einer Fachsprache ausmacht. Diese starke Betonung des lexikalischen Aspekts führte dazu, dass das Wesen der Fachsprachen fast ausschließlich in ihrer Terminologien gesucht wurde (Hahn 1981:11). Die Begriffe Fachsprache und wortschatz wurden eine zeitlang als Synonyme verwendet. Auch heute ist die Terminologiearbeit ein wichtigstes Gebiet der Fachsprachenforschung, sie wird aber eher als Ergänzung der textlinguistischen Analyse des fachsprachlichen Materials betrachtet.

\section{Die Textsorte - Definition des Begriffes}

Wenn wir von Textsorten sprechen, meinen wir meistens Klassen von Texten, die sich durch bestimmte Merkmale auszeichnen. Ich werde mich für weitere Überlegungen zum Thema Textsorte der Definition von Brinker (1988:123) bedienen, der die Textsorte folgendermaßen definiert:

DIE TEXTSORTEN sind konventionell geltende Muster für komplexe sprachliche Handlungen und lassen sich als jeweils typische Verbindungen von kontextuellen (situativen), kommunikativ-funktionalen und strukturellen (grammatischen und thematischen) Merkmalen beschreiben. Sie haben sich in der Sprachgemeinschaft historisch entwickelt und gehören zum Alltagswissen der Sprachteilhaber; sie besitzen zwar eine normierende Wirkung, erleichtern aber zugleich den kommunikativen Umgang, indem sie den Kommunizierenden mehr oder weniger feste Orientierungen für die Produktion und Rezeption von Texten geben. 
Man könnte diese Definition lediglich um die Feststellung ergänzen, dass die Textsorte nicht nur eine gewisse Orientierung den Kommunizierenden für Textproduktion und -rezeption geben, sondern dass die Beschreibung von ihren Merkmalen gleichzeitig eine wesentliche Hilfe für den Übersetzungsprozess bildet. Die Übersetzer, die sich über typische Merkmale einer Textsorte informieren, werden auf bestimmte Erscheinungen aufmerksam und vermeiden dadurch bestimmte Übersetzungsfehler.

\section{Einige Kriterien der Textsortenbestimmung}

Als Ausgangspunkt für Klassifikationsversuche der verschiedenen Textsorten kann das „Organon Modell“ von Karl Bühler (1965:34) dienen. Das Modell beschreibt die Funktionsweise von Sprachzeichen im Rahmen konkreter Kommunikationszusammenhänge. In diesem Sinne werden den Sprachzeichen drei mögliche Grundfunktionen zugeschrieben:

- die Darstellungsfunktion

- die Ausdrucksfunktion

- die Appellsfunktion

Die von Bühler herausgearbeiteten drei Grundfunktionen schaffen einen wichtigen Zugang zu einer sprachwissenschaftlich begründeten Texttypologie (vgl. Studienbuch Linguistik 1996:246). Eine genauere Beschreibung der Textsorten, die nach dieser Typologie klassifiziert werden können, hat K. Reiß (1971) in ihren Arbeiten unternommen. Grundsätzlich kommen zwar in jedem Text alle drei Funktionen der Sprache ins Spiel, doch lässt sich je nach dem Übergewicht der einen oder anderen Funktion in einem gegebenen Text eine Unterscheidung von drei Grundtypen rechtfertigen:

a) der Darstellungsfunktion der Sprache gemäß - inhaltsbetonte Texte

b) der Ausdrucksfunktion gemäß - formbetonte Texte

c) der Appellfunktion gemäß - appellbetonte Texte

Der Versuch, die in der Praxis gegebene Vielfalt von Texten diesen drei Grundtypen zuzuordnen, muss davon ausgehen, dass sich jeder Texttyp je nach Maßgabe der dargelegten Merkmale wiederum in eine Vielzahl von Textarten auffächert. Die Werbetexte technischer Unternehmen, die hier analysiert werden, gehören nach der oben genannten Klassifikation zu einer Mischgruppe, weil in diesen Texten sowohl die Darstellungsfunktion als auch die Appellfunktion der Sprache eine wichtige Rolle spielen. Die Darstellungsfunktion verstanden als ästhetische Wirkung, die auf dem Empfänger ausgeübt werden soll, spielt eine untergeordne- 
te Rolle in pragmatischen Texten. Die Prospekte technischer Unternehmen gehören jedoch zu Werbetexten, die in einer bestimmten äußeren Form angeboten werden müssen. Bei einer Vielfalt von fast identischen Produkten auf dem Markt, derer technische Parameter völlig vergleichbar sind, kann manchmal die äußere Form, in der die Ware präsentiert wird ausschlaggebend sein.

Die auf sprachwissenschaftlicher Grundlage erarbeitete Klassifikation von Texttypen scheint jedoch nicht ausreichend zu sein, weil sie keine Informationen über kommunikative Funktionen eines Textes vermittelt. Die kommunikative Funktion kann erst nach der Analyse der Faktoren bestimmt werden, die eine kommunikative Situation bilden (vgl. Nord 1995:49). Diese Faktoren werden auch als textexterne Faktoren bezeichnet im Gegensatz zu den textinternen, die sich auf den Textinhalt beziehen. Diese Textklassifikationsfaktoren (Studienbuch Linguistik 1996:251) lassen sich graphisch folgendermaßen darstellen:

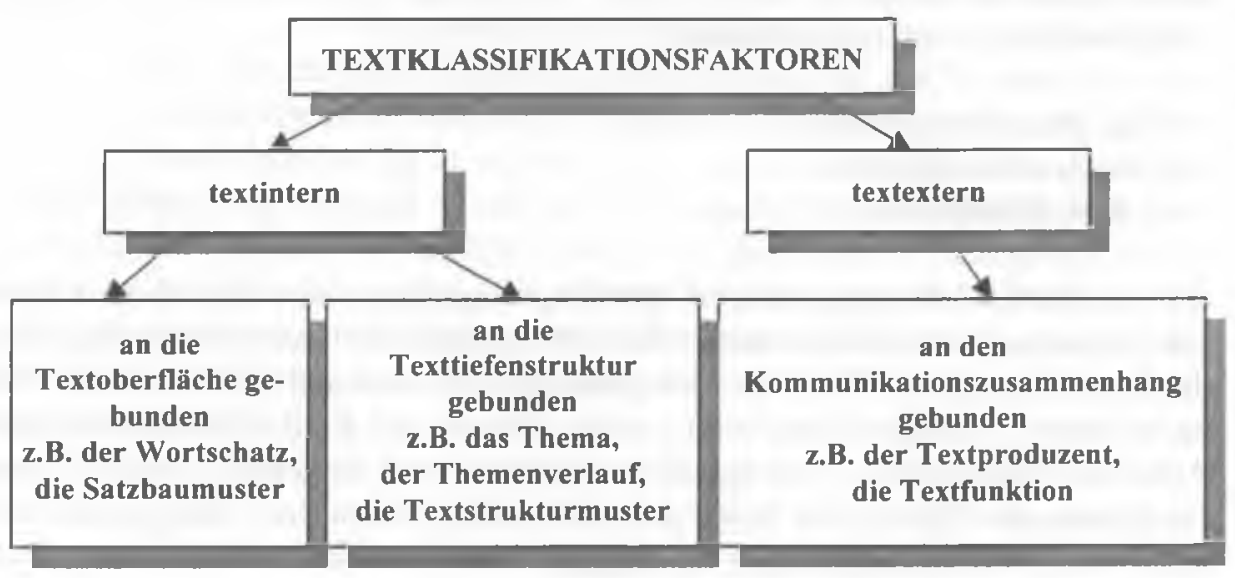

Abb. 1. Textklassifikationsfaktoren

\section{Die textinternen Faktoren in Werbetexten technischer Unternehmen}

Wichtige Gruppe von Merkmalen, die uns erlauben, einen bestimmten Text einer Textgattung zuzuordnen, sind die textinternen Faktoren sowohl an der Textoberfläche als auch in seinen Tiefenstrukturen.

Wir möchten einige dieser Merkmale im Hinblick auf die technischen Werbeprospekte hier besprechen. Gleichzeitig werden diese Merkmale ebenfalls am Beispiel des Prospektes der Firma Wacker Chemie GmbH dargestellt. 
Graphische Ebene: Die graphische Gestaltung aller Prospekte sieht ähnlich aus. Wir haben normalerweise mit ein paar mit technischen Informationen gedruckten Seiten zu tun, die oft von Photos und Schemata begleitet werden. Die Bilder spielen in dieser Textgattung keine Anziehungsrolle, sondern stellen die Produkte objektiv dar und wirken abwechslungsreich im Gegensatz zu dem mit technischen Daten überfüllten Text. Wichtiger als die Bilder sind die Tabellen und Abbildungen, in denen oft wichtige Informationen enthalten sind. In dem beigelegten Prospekt können wir sowohl Photos von Waren aus Kautschuk sehen, als auch Schemata und chemische Formeln. Die graphische Darstellung der Informationen unterstützt hier die Intention des Senders, weil die Produkte sachlich und transparent präsentiert werden und der Empfänger durch die graphische Form nicht abgelenkt wird.

Wortwahl: Die Wortwahl dient als eine sehr wichtige Informationsquelle für Textsortenbestimmung, anhand des benutzten Wortschatzes können wir feststellen, an welche Empfängergruppe sich der Sender gerade wendet. Für Werbetexte technischer Unternehmen ist eine Terminologisierung und Verwendung von fachsprachlichem Wortschatz charakteristisch. Diese Tendenz lässt sich unabhängig von der Originalsprache der Prospekte feststellen und bildet eine wichtige Herausforderung für den Übersetzer, der auch in der Zielsprache eine äquivalente Wortwahl treffen muss.

Der Prospekt von der Firma Wacker Chemie GmbH enthält fachsprachliche Informationen aus dem Bereich Chemie. Neben der Terminologisierung kann man in diesem Prospekt bezüglich der Wortwahl noch ein Phänomen bemerken. Es sind die Adjektive, mit denen die Produkteigenschaften beschrieben wurden (spezielle, hervorragende, problemlose usw.). Die verwendeten Adjektive haben nur positive Assoziationen und unterstützen die Senderintention.

Art und Häufigkeit von Satzbaumustern: Die häufigsten Satzbaumuster für Werbetexte technischer Unternehmen sind Nominalkonstruktionen, Partizipialgefüge, wie auch Passivkonstruktionen, die in einem fachlichen Text Eindruck einer Distanzierung und Sachlichkeit schaffen sollen. Wir finden viele solche Konstruktionen in dem Prospekt der Firma Wacker Chemie GmbH (z.B. problemlose, wirtschaftliche Verarbeitbarkeit oder eine große Palette farblicher Gestaltungsmöglichkeiten).

Themenbildung und Themenablauf: In bezug auf diese beiden Kriterien stellen wir an fachliche Werbetexte fast immer die Anforderung, dass das Thema in einzelnen Punkten vertiefend behandelt wird. Wir erwarten Übermittlung der Sachinformationen zu einem konkreten Thema. In dem analysierten Prospekt werden wir über die wichtigsten Eigenschaften des Kautschuks informiert, z.B. über mögliche Temperaturbereiche (von $-50^{\circ} \mathrm{C}$ bis $z u+200^{\circ} \mathrm{C}$ ) in denen das Material sein Einsatz findet. 
Thema: Das eigentliche Thema können wir manchmal schon der Textsortenbenennung entnehmen. Sonst werden wir darüber in den fettgedruckten Überschriften informiert. So ist auch bei diesem Prospekt. Der Text wurde mit einer großen Überschrift versehen: Elastosil $₫ R$ und unten, ebenfalls fettgedruckt: Der Kautschuk für hohe Anforderungen: Silikonkautschuk Elastosil@R. Der Textempfänger erhält also noch vor dem Lesen einige Informationen über das Thema des Textes.

Textstrukturmuster: Mit dem Textstrukturmuster werden die spezifische Gliederungs- oder Baustruktur gemeint, die oft als Makrostruktur bezeichnet wird (Van Dijk 1980:49) Die Makrostruktur repräsentiert die „globale Bedeutung“ eines Textes, die durch Verfahren der parahrasierenden Reduktion gewonnen wird. Es handelt sich dabei um Auslassen, Verallgemeinern und Konstituieren. Das Ergebnis ist eine Textzusammenfassung, die als direkte Verbalisierung der Makrostruktur aufgefasst wird. Nach dieser Auffassung sollen zu einer Textsorte alle Prospekte technischer Unternehmen gehören, die eine ähnliche Makrostruktur und ähnliche "globale Bedeutung" haben. Die Makrostruktur und die "globale Bedeutung“ des Textes Elastosil $® R$ entspricht ebenfalls diesem Muster.

\section{Die textexternen Faktoren in Werbetexten technischer Unternehmen}

An dieser Stelle werden am Beispiel vom Prospekt der Firma Wacker Chemie GmbH detaillierter die Faktoren besprechen, die in den Werbematerialien technischer Unternehmen die kommunikative Situation bilden. Es handelt sich hier um die textexternen Faktoren.

Textsender/Textproduzent: Als Sender eines Textes wird normalerweise eine Person oder Institution bezeichnet, die den Text zu einer Mitteilung an jemand anderen verwendet bzw. mit ihm etwas erreichen will. Als Textproduzent kann man dagegen eine Person oder Institution verstehen, die gemäß den Konventionen und Vertextungsnormen den jeweiligen Text nach Maßgaben des Senders produziert. Informationen darüber, ob der Sender und der Textproduzent dieselbe Person ist, lassen sich in den meisten Fällen dem Textumfeld (z.B. Quellenangabe, Überschrift, Adresse der Firma) entnehmen. Der Sender eines Prospektes ist normalerweise eine Firma, die über ihre Produkte oder Dienstleistungen informieren möchte. Die Angaben über den Sender vom Prospekt der Firma Wacker Chemie GmbH befinden sich sowohl im Textumfeld (Überschrift, Adresse), wie auch im eigentlichen Text. Wir erfahren daraus, dass der Sender eine deutsche Firma ist, die Kautschuke herstellt. Der Name des Senders erscheint in dem Text und seinem Umfeld nicht, was darauf hinweist, dass seine Rolle untergeordnet ist. 
Intention: Die Frage nach der Intention des Senders besitzt für die Texttypologie besondere Bedeutung: Was will er mit dem Text bewirken? Wozu will er den Empfänger anregen? Die Intention des Senders hat wesentlichen Einfluss auf die Textgestaltung: das Thema, den Inhalts- und Informationsauswahl, wie auch auf die Form (z.B. Aufbau, stilistische Mittel, Zitierweise, Einsatz nonverbaler Gestaltungsmittel etc.), durch die dann weiter ein wichtiger Hinweis auf die Textsorte gegeben wird. Die Informationen über die Intention des Textverfassers können uns auch beim Identifizieren der textexternen Faktoren wie Empfänger, Medium und Textfunktion sowie der textinternen Merkmale wie Textaufbau behilflich sein.

Gerade die Intention des Senders besitzt für Prospekte technischer Unternehmen große Bedeutung. Der Sender möchte hier den Empfänger nicht nur über seine Produkte informieren, sondern er möchte ihn gleichzeitig auch zu dem Kauf bewegen. Diesem Ziel werden alle sprachlichen Mittel untergeordnet, die den gesamten Text bilden. Die Intention des Senders ist in jedem Prospekt klar. Die potentiellen Kunden sollen beeinflusst werden. Aus diesem Grunde wird die Werbung oft als „programmiertes Informieren“ bezeichnet (vgl. Skowronek 1993:5), was sehr gut die Natur technischer Werbetexten beschreibt.

Der Textverfasser wählt für jeden Text angenommenen bzw. konventionalisierten Vertextungsformen. Der Rezipient sollte sofort an den verwendeten Vertextungsformen erkennen, welche Intention der Textsender hatte. Man muss hier betonen, dass den Sender eines Textes nicht nur eine Intention leiten muss, sondern dass unterschiedliche Intentionen in verschiedener Gewichtung kombiniert auftreten können.

In dem Prospekt der Firma Wacker Chemie GmbH ist die Intention des Senders auch leicht erkennbar. Die schwarzgedruckte Überschrift: „Der Kautschuk für hohe Anforderungen“ soll den Empfänger überzeugen, dass er mit einem Produkt von hoher Qualität zu tun hat, das seinen Erwartungen bestimmt entspricht.

Empfänger: Mit Empfänger ist der Hörer oder der Leser des Textes gemeint. Der Sender muss zunächst über gewisse Informationen über den Empfänger verfügen. Die Determinanten des Empfängers: Alter, Geschlecht, Bildungsstand, gesellschaftliches Umfeld, geographische Herkunft, etc. sind wichtig, weil sie dem Sender helfen, den Leser erfolgreich anzusprechen. Besonders wichtig sind die Wissensvoraussetzungen des Empfängers, deren richtige Einschätzung dem Sender erlaubt, bestimmte Informationen als bekannt vorauszusetzen und verknappt wiederzugeben und andere ausführlich zu vermitteln, so dass der Leser weder über- noch unterfordert wird (vgl. Nord 1995:65). Da wir über die Kommunikation nicht sprechen können, wenn kein Verständigungswille seitens des Empfängers vorhanden ist, müssen wir eine Rezeptionsabsicht beim Empfänger annehmen. Davon zu unterscheiden ist einerseits die Erwartung, die zum Bereich der Wissensvoraussetzungen gehört, und andererseits die Reaktion des Empfängers auf 
den Text, die sich nach der Rezeption einstellt und damit zur Wirkung des Textes gehört.

Die Aufschlüsse über die Empfängerdaten können wir, wie es auch bei den Senderdaten der Fall war, den textinternen Merkmalen und dem Textumfeld entnehmen oder sie ergeben sich aus den Informationen zum Sender und seiner Intention sowie aus den Faktoren wie Medium, Ort, Zeit und Anlass ergeben. Dabei sind standardisierten Textsorten meist auch standardisierte Empfängererwartungen zuzuordnen. Den Empfängerkreis von Prospekten technischer Unternehmen bilden sowohl Fachleute wie auch interessierte Laien, die dem Prospekt ein Minimum des Fachwissens entnehmen möchten.

Bei dem Prospekt der Firma Wacker Chemie GmbH haben wir mit zwei potentiellen Empfängergruppen zu tun. Einerseits sind es Fachleute aus dem Bereich Gummiartikel, die dem Text bestimmte technische Informationen entnehmen möchten, andererseits können es auch Mitarbeiter von Firmen sein, die für den Materialeinkauf zuständig sind. Gerade für die letzte Kundengruppe ist die Appellfunktion des Textes von Bedeutung. Heutzutage haben die Firmen mit einer großen Warenpalette zu tun und die Prospekte sollen ihnen Hilfe bei der Entscheidung leisten und sie gleichzeitig zum Kauf veranlassen.

Medium/Kanal: $\mathrm{Zu}$ weiteren textexternen Faktoren, die der Textanalyse dienen, gehört das Medium, oder anders gesagt der Kanal, über den sich der Sender an den Rezipienten wendet.

Ch. Nord (1995:64) versteht unter diesem Begriff das Transportmittel, mit dessen Hilfe der Text an den Empfänger gelangt. Dabei sind die technischen Einzelheiten weniger von Interesse als das Problem der Wahrnehmbarkeit und der Speicherung. Es geht hier darum, ob der Text zum Empfänger mündlich oder schriftlich transportiert wird.

Der Faktor Medium ist wichtig, weil er darüber entscheidet, wie groß der Adressatenkreis sein kann. Ein kurzer Werbetext in einer Fachzeitung für Architekten hat bestimmt weniger Empfänger als ein interessanter Prospekt, der in vielen Tausend Exemplaren herausgegeben und während einer Baufachmesse verteilt wird. Die Zahl der Leser eines Prospektes kann sich manchmal auf die Anzahl der Käufer eines Produktes begrenzen, wobei diese weiter um Fachleute begrenzt sein könnte, die Informationen nicht unbedingt einem Prospekt entnehmen müssen, weil sie z.B. ein persönliches Treffen mit dem Produzenten oder seinem Vertreter bevorzugen. Diese Zahl wird jedoch größer, wenn wir an die potentiellen Kunden denken, die sich nur aus Informationsgründen mit dem Werbematerial bekannt machen wollen. Bei dem Faktor Medium muss man unbedingt das Internet erwähnen, den neuesten Weg der Informationsübermittlung. Heutzutage haben wir mit einem sehr großen Interesse für diesen Bereich zu tun. Die fachsprachlichen Informationen werden entweder per e-mail geschickt oder von WWW-Seiten abgerufen und gelangen dadurch an viel mehr Empfänger als je zuvor. Die in dem Netz angebotenen Informationen unterscheiden sich auch in ih- 
rer sprachlichen Form von den Informationen, die in traditionellen Kanälen präsentiert werden.

Das von der Firma Wacker Chemie GmbH gewählte Medium hat die Form einer technischen Broschüre, die in ein paar Tausend Exemplaren herausgegeben wurde. Der Prospekt gelangt an die Kunden per Post oder wird von Vertretern der Firma den Kunden persönlich überreicht. Die Prospekte können auch während einer Fachmesse an die vorbeigehenden Besucher verteilt werden.

Kommunikationsanlass: Mit Kommunikationsanlass werden meistens die Gründe gemeint, aus denen ein Text geschrieben oder geschickt wird. Nicht bei allen Texten ist der Anlass leicht identifizierbar. Bei den Kauf- oder Verkaufanzeigen z.B. ist der Anlass relativ leicht zu erkennen: es ist die Tatsache, dass der Textsender etwas entweder kaufen oder verkaufen will. Der Anlass für die Produktion eines Textes gibt Hinweise auf die Senderintention und auf die Textfunktion. In den Werbetexten technischer Unternehmen ist der Anlass meistens nur schwer erkennbar, bis auf die Fälle, wenn wir mit einem neuen Produkt zu tun haben, das dem Markt vorgestellt werden soll, oder mit Prospekten, die für bestimmte Anlässe, wie z.B. Messe oder Jahrestag herausgegeben werden. Der Kommunikationsanlass für den hier analysierten Prospekt lässt sich nicht eindeutig bestimmen.

Textfunktion: Die Anhaltspunkte, die auf den konkreten Anlass der Textproduktion verweisen, liefert besonders die Textfunktion, wenn sie durch eindeutige Präsignale, wie z.B. Textsortenbezeichnung (Gebrauchsanweisung, Protokoll, Trauerrede etc.) angezeigt wird.

Die Textfunktion ist die kommunikative Funktion bzw. Kombination der kommunikativen Funktionen eines Textes in einer konkreten Situation. K. Brinker (1988:77) definiert sie als der Sinn, den ein Text in einem Kommunikationsprozess erhält bzw. als der Zweck, den ein Text im Rahmen einer Kommunikationssituation erfüllt. Ein Text kann mehr als eine kommunikative Funktion besitzen. So haben z.B. die fachlichen Werbetexte gleichzeitig eine informative und eine appellative Funktion. Die letzte wird häufig bei den Prospekten, direkt durch bestimmte sprachliche Strukturen, wie z.B. Formen des Imperativs, Höflichkeitsformeln, Nominalisierungen oder Infinitivkonstruktionen angezeigt. Diese sprachliche Strukturen befinden sich auch in dem hier analysierten Prospekt. Die sachliche Beschreibung der Anwendungsmöglichkeiten und Eigenschaften des Kautschuks weist auf die informative Funktion des Textes hin.

\section{Schlussfolgerungen}

Die Liste der hier aufgezeigten Kriterien oder Merkmale, die uns eine genaue Textklassifikation erlauben, ist bestimmt nicht vollständig. Obwohl es alltagssprachlich relativ leicht ist, einzelnen Textexemplaren eine Textsortenbezeichnung zuzuordnen, ist es im Rahmen textlinguistischer Forschung bisher nicht ge- 
lungen, eine einheitliche, für alle Fälle „gültige“ Klassifikation der Texte in bestimmte Textsorten zu erstellen (vgl. Studienbuch Linguistik 1996:248).

Die Versuche, eine einheitliche Textsortenbestimmung zu schaffen, bleiben jedoch nach wie vor Hauptinteresse der übersetzungsorientierten linguistischen Textanalyse, weil die Textgattung, der ein bestimmter Text gehört, oft als Hauptfaktor bezeichnet wird, der Kriterien wie Übersetzungsprinzipen und -verfahren bestimmt.

Die bisherigen Fachtextuntersuchungen haben deutlich gemacht, dass im Hinblick auf eine überzeugende Klassifikation von Fachtexten noch vielfältig Probleme zu lösen sind. Eine umfassende Differenzierung von Fachtexten kann aus der Sicht der Fremdsprachenforschung und der Textlinguistik nicht erreicht werden, da dafür ein interdisziplinäres Zusammengehen mehrerer linguistischen Disziplinen erforderlich ist. Um ein komplexes Bild des Gegenstandes zu zeichnen, müssen nach dem Konzept von Baumann (1986:17) folgende Betrachtungsebenen und Disziplinen mitberücksichtigt werden:

- die soziale Dimension (Soziolinguistik)

- die gedankliche Dimension (Sprachtheorie)

- die funktional-kommunikative Dimension

- (funktional-kommunikative Sprachbetrachtung)

- die textuelle Dimension (Textlinguistik)

- die stilistische Dimension (Stilistik)

- die semantische Dimension (Semantik)

Die Untersuchung von Fachtextsorten ist ein wichtiger Forschungsbereich in der gegenwärtigen Textlinguistik. Die Ergebnisse dieser Forschung bereichern unser Wissensstand über Art und Weise von Textensklassifikation und finden ihre Verwendung in der Übersetzerausbildung und Fachsprachendidaktik.

Der hier durchgeführte Versuch, Textsortenkriterien für Werbematerialien technischer Unternehmen zu erarbeiten, ist nur ein Teil einer umfangreicheren Untersuchung der Textsortenproblematik im Bereich der technischen Fachsprachen.

\section{LITERATUR}

Baumann, K.D. (1986): Ein integrativer Ansatz zur Analyse von Fachkommunikation. (Diss. B) Leipzig. In: Pfeiffer, W. (Hrsg.): Deutsch als Fachsprache in der Deutschlehrerausbildung und -Fortbildung. Poznań: Wydawnictwo Naukowe UAM.

Baumann, K.D. (1990): Der aktuelle Entwicklungsstand der Fachsprachenforschung - ein Überblick. In: Pfeiffer, W. (Hrsg.): Deutsch als Fachsprache in der Deutschlehrerausbildung und Fortbildung. Poznan: Wydawnictwo Naukowe UAM, 9-25.

Brinker, K. (1988): Linguistische Textanalyse. Berlin: Schmidt.

Bühler, K. (1965): Sprachtheorie, 2. Auflage. Stuttgart.

Hahn, W. (1981): Fachsprachen. Darmstadt. In: Pfeiffer, W. (Hrsg.): Deutsch als Fachsprache in der Deutschlehrerausbildung und-Fortbildung. Poznań: Wydawnictwo Naukowe UAM. 
Linke, A.; Nussbaumer, M.; Portmann, P. (1996): Studienbuch Linguistik. Tübingen: Max Niemeyer. Nord, Ch. (1995): Textanalyse und Übersetzen. Heidelberg: Verlag.

Reiß, K. (1971): Möglichkeiten und Grenzen der Übersetzung. München: Max Hueber.

Skowronek, K. (1993): Reklama - Studium pragmalingwistyczne. Kraków: PAN.

Van Dijk, T.A. (1980): Textwissenschaft. München: DTV.

\begin{abstract}
ANLAGE
Kopie des Prospekts „ElastosilßR” von der Firma Wacker Chemie GmbH - ein Beispiel vom Textkorpus, der vom 01.10.1999 bis 01.06.2000 gesammelt wurde.
\end{abstract}

